PROCEEDINGS OF THE

AMERICAN MATHEMATICAL SOCIETY

Volume 133, Number 6, Pages 1769-1775

S 0002-9939(05)07830-5

Article electronically published on January 14, 2005

\title{
WEAK WEIGHTED INEQUALITIES FOR A DYADIC ONE-SIDED MAXIMAL FUNCTION IN $\mathbb{R}^{n}$
}

\author{
SHELDY OMBROSI
}

(Communicated by Andreas Seeger)

\begin{abstract}
In this note we introduce a dyadic one-sided maximal function defined as

$$
M^{+, d} f(x)=\sup _{Q \text { dyadic: } x \in Q} \frac{1}{|Q|} \int_{Q^{+}}|f|,
$$

where $Q^{+}$is a certain cube associated with the dyadic cube $Q$ and $f \in$ $L_{\text {loc }}^{1}\left(\mathbb{R}^{n}\right)$. We characterize the pair of weights $(w, v)$ for which the maximal operator $M^{+, d}$ applies $L^{p}(v)$ into weak- $L^{p}(w)$ for $1 \leq p<\infty$.
\end{abstract}

\section{INTRODUCTION, NOTATION AND MAIN RESULTS}

In [10] E. Sawyer introduced the class of weights $A_{p}^{+}$, which characterizes the pair of weights $(w, v)$ such that the one-sided Hardy-Littlewood maximal operator applies $L^{p}(v)$ into weak- $L^{p}(w)$. These classes of weights and their associated theory have been the subject of much study. Different proofs of Sawyer's results and more general extensions are given in the papers of F. J. Martín-Reyes, Ortega-Salvador and A. de la Torre [6], [3] and [5. In [1] H. Aimar, L. Forzani and F. J. MartínReyes proved the existence of one-sided singular integral operators, and L. de Rosa and C. Segovia have developed a great part of the theory of one-sided weighted Hardy spaces (see 8] and [9]). However, as far as the author knows, the theory of one-sided weights has been developed only in $\mathbb{R}$.

The results in this note try to give a first step to overcome that limitation. We will define a dyadic one-sided maximal function in $\mathbb{R}^{n}$, and we characterize the pair of weights $(w, v)$ such that the operator associated with this maximal function applies $L^{p}(v)$ into weak- $L^{p}(w)$ (Theorem 1.2 below).

If $I=[a, b)$ is a bounded interval we denote $I^{+}=[b, 2 b-a)$ and $I^{-}=[2 a-b, a)$. If $Q=I_{1} \times I_{2} \times \ldots \times I_{n}$ is a cube in $\mathbb{R}^{n}$, we denote $Q^{+}=I_{1}^{+} \times I_{2}^{+} \times \ldots \times I_{n}^{+}$and $Q^{-}=I_{1}^{-} \times I_{2}^{-} \times \ldots \times I_{n}^{-}$.

Given $f \in L_{l o c}^{1}\left(\mathbb{R}^{n}\right)$, we define the dyadic one-sided maximal function $M^{+, d} f(x)$ as

$$
M^{+, d} f(x)=\sup _{Q \text { dyadic: } x \in Q} \frac{1}{|Q|} \int_{Q^{+}}|f| .
$$

Received by the editors February 19, 2004.

2000 Mathematics Subject Classification. Primary 42B25; Secondary 28 B99.

Key words and phrases. Weights, dyadic one-sided maximal function.

(C)2005 American Mathematical Society Reverts to public domain 28 years from publication 
In a similar way $M^{-, d} f(x)=\sup _{Q \text { dyadic: } x \in Q} \frac{1}{|Q|} \int_{Q^{-}}|f|$. In dimension one, this maximal function and, more generally, dyadic one-sided fractional maximal functions were defined in $[5]$.

Let $x \in \mathbb{R}^{n}, x=\left(x_{1}, x_{2}, \ldots, x_{n}\right)$, and let $h$ be a positive real number. We denote $Q_{x, h}=\left[x_{1}, x_{1}+h\right) \times\left[x_{2}, x_{2}+h\right) \times \ldots \times\left[x_{n}, x_{n}+h\right), Q_{x, h^{-}}=\left[x_{1}-h, x_{1}\right) \times\left[x_{2}-h, x_{2}\right) \times$ $\ldots \times\left[x_{n}-h, x_{n}\right)$, and $Q_{x, h}^{\frac{1}{2}}=\left[x_{1}+\frac{h}{2}, x_{1}+h\right) \times\left[x_{2}+\frac{h}{2}, x_{2}+h\right) \times \ldots \times\left[x_{n}+\frac{h}{2}, x_{n}+h\right)$. With this notation, we define the following maximal functions:

$$
M^{+} f(x)=\sup _{h>0} \frac{1}{\left|Q_{x, h}\right|} \int_{Q_{x, h}}|f|, M^{-} f(x)=\sup _{h>0} \frac{1}{\left|Q_{x, h^{-}}\right|} \int_{Q_{x, h^{-}}}|f|,
$$

and

$$
N^{+} f(x)=\sup _{h>0} \frac{1}{\left|Q_{x, h}^{\frac{1}{2}}\right|} \int_{Q_{x, h}^{\frac{1}{2}}}|f|
$$

In dimension $n=1$, the maximal functions $M^{+, d} f(x), N^{+} f(x)$, and $M^{+} f(x)$ are pointwise equivalents (see [5]), this fact is not true if $n>1$.

Let $Q=\left[a_{1}, b_{1}\right) \times\left[a_{2}, b_{2}\right) \times \ldots \times\left[a_{n}, b_{n}\right)$, with $b_{i}-a_{i}=h$, and let $s$ be a positive real number. We denote $(Q)^{s,+}=\left[a_{1}, a_{1}+s h\right) \times\left[a_{2}, a_{2}+s h\right) \times \ldots \times\left[a_{n}, a_{n}+s h\right)$, and $(Q)^{s,-}=\left[b_{1}-s h, b_{1}\right) \times\left[b_{2}-s h, b_{2}\right) \times \ldots \times\left[b_{n}-s h, b_{n}\right)$. We observe that $\left|(Q)^{s,+}\right|=\left|(Q)^{s,-}\right|=s^{n}|Q|$ and $Q=(Q)^{1,+}=(Q)^{1,-}$.

Remark 1.1. Let $R$ and $Q$ be cubes such that $R^{+} \subset Q^{+}$. Then $R \subset(Q)^{2,+}$.

As usual, a weight $w(x)$ is a measurable and non-negative function. If $E \subset \mathbb{R}^{n}$ is a Lebesgue measurable set, we denote its $w$-measure by $w(E)=\int_{E} w(t) d t$.

We will say that a pair of weights $(w, v)$ satisfies the condition $A_{p}^{+}\left(\mathbb{R}^{n}\right)$ (or $(w, v)$ belongs to the class $\left.A_{p}^{+}\left(\mathbb{R}^{n}\right)\right), p>1$, if there exists a constant $C$ such that for every cube $Q$,

$$
\int_{Q} w\left(\int_{Q^{+}} v^{-\frac{1}{p-1}}\right)^{p-1} \leq C|Q|^{p}
$$

The condition $A_{1}^{+}\left(\mathbb{R}^{n}\right)$ is given by

$$
M^{-} w(x) \leq C v(x) \text { a.e. }
$$

In 4] F. J. Martin-Reyes obtained interesting geometric properties of these classes of weights.

We define similar classes of weights on dyadic cubes. We will say that a pair of weights $(w, v)$ satisfies the condition $A_{p}^{+, d}\left(\mathbb{R}^{n}\right)(p>1)$ if (1) holds for every dyadic cube $Q$, and the condition $A_{1}^{+, d}$ is given by $M^{-, d} w(x) \leq C v(x)$ a.e.

It is clear that if $w \geq 0$ is a nondecreasing function in each coordinate, the pair $(w, w)$ satisfies the condition $A_{p}^{+}\left(\mathbb{R}^{n}\right)$ and therefore $A_{p}^{+, d}\left(\mathbb{R}^{n}\right)$. Thus, these conditions are weaker than the Muckenhoupt's condition $A_{p}\left(\mathbb{R}^{n}\right)$ introduced in [7], which characterizes the pair of weights such that the Hardy-Littlewood maximal operator applies $L^{p}(v)$ into weak- $L^{p}(w)$. 
The next theorem solves a conjecture of A. de la Torre and F. J. Martín-Reyes.

Theorem 1.2. Let $w$ and $v$ be weights on $\mathbb{R}^{n}$ and let $1 \leq p<\infty$. Then, the following conditions are equivalent:

(a) The pair of weights $(w, v)$ satisfies the condition $A_{p}^{+, d}\left(\mathbb{R}^{n}\right)$.

(b) The maximal operator $M^{+, d}$ is of weak type $(p, p)$ with respect to the pair $(w, v)$, that is: there is a constant $C$ such that for every function $f \in$ $L_{\text {loc }}^{1}\left(\mathbb{R}^{n}\right)$ and every $\lambda>0$,

$$
w\left(\left\{x: M^{+, d} f(x)>\lambda\right\}\right) \leq C \lambda^{-p} \int_{\mathbb{R}^{n}}|f|^{p} v .
$$

Theorem 1.3. Let $w$ and $v$ be two weights on $\mathbb{R}^{n}$ and let $1 \leq p<\infty$. If $(w, v)$ belongs to the class $A_{p}^{+}\left(\mathbb{R}^{n}\right)$, then the maximal operator $N^{+} f$ is of weak type $(p, p)$ with respect to the pair $(w, v)$.

\section{Proof of the Results}

Let $f$ be an integrable and non-negative function and let $\lambda$ be a positive real number. We denote $\Omega_{\lambda}=\left\{x: M^{+, d} f(x)>\lambda\right\}$. It is not hard to see that there exists a disjoint collection of dyadic cubes $\left\{Q_{j}\right\}$ such that

$$
\bigcup_{j} Q_{j}=\Omega_{\lambda} \text { and for each } j, f_{Q_{j}^{+}}=\frac{1}{\left|Q_{j}\right|} \int_{Q_{j}^{+}} f>\lambda \text {. }
$$

In general, the associated collection $\left\{Q_{j}^{+}\right\}$is not disjoint. This fact is the main difficulty appearing in our proof of Theorem 1.2. However with the next lemma we overcome that difficulty.

Lemma 2.1. Let $1 \leq p<\infty$. Let $(w, v)$ be a pair of weights satisfying the condition $A_{p}^{+, d}\left(\mathbb{R}^{n}\right)$. Let $\mu$ be a positive real number. If $\left\{Q_{j}\right\}_{j \in \Gamma_{\mu}}$ is a disjoint collection of dyadic cubes $\left(\Gamma_{\mu}\right.$ denotes the family of indexes $j$ ) such that for each $j \in \Gamma_{\mu}, \mu<$ $f_{Q_{j}^{+}} \leq 2 \mu$, then

$$
\sum_{j \in \Gamma_{\mu}} w\left(Q_{j}\right) \leq \frac{2^{p+n+2} C}{\mu^{p}} \int_{\cup_{j \in \Gamma_{\mu}} Q_{j}^{+}}|f|^{p} v,
$$

where $C$ is the smallest constant that we can take in the condition $A_{p}^{+, d}\left(\mathbb{R}^{n}\right)$ for the pair $(w, v)$.

Proof. For each integer $m \geq 0$, we define the subfamily of indexes $i_{m}$ in $\Gamma_{\mu}$ as

$i_{m}=\left\{j \in \Gamma_{\mu}:\right.$ there exist exactly $m$ cubes $Q_{s}^{+}: Q_{j}^{+} \varsubsetneqq Q_{s}^{+}$with $\left.s \in \Gamma_{\mu}\right\}$, and we denote

$$
\sigma_{m}=\bigcup_{j \in i_{m}} Q_{j}^{+}
$$

Remarks. (1) It is clear that $\Gamma_{\mu}=\bigcup_{m \geq 0} i_{m}$. Furthermore, if $j_{1}, j_{2}$ belong to $i_{m}$, then $Q_{j_{1}}^{+} \cap Q_{j_{2}}^{+}=\emptyset$, and therefore

$$
\sum_{j \in i_{m}} \int_{Q_{j}^{+}} f=\int_{\sigma_{m}} f
$$

(2) If $j \in i_{m+1}(m \geq 0)$, then for each integer $k, 0 \leq k \leq m$, there is only one $j_{k} \in i_{k}$ such that $Q_{j}^{+} \subset Q_{j_{k}}^{+}$. Thus $\sigma_{m+1} \subset \sigma_{m}$ and $\int_{\sigma_{m+1}} f \leq \int_{\sigma_{m}} f$. 
(3) We fix a non-negative integer $m_{0}$ and $j_{0} \in i_{m_{0}}$. If $Q_{j}^{+} \varsubsetneqq Q_{j_{0}}^{+}$, then $j \in i_{m}$ where $m>m_{0}$, and moreover, by Remark 1.1 we have that $Q_{j} \subset\left(Q_{j_{0}}\right)^{2,+}$.

Taking into account the last observation and since the cubes $Q_{j}$ are disjoint we have

and

$$
\bigcup_{m>m_{0}} \bigcup_{\substack{j \in i_{m}: \\ Q_{j}^{+} \varsubsetneqq Q_{j_{0}}^{+}}} Q_{j} \subset\left(Q_{j_{0}}\right)^{2,+}
$$

$$
\sum_{m>m_{0}} \sum_{\substack{j \in i_{m}: \\ Q_{j}^{+} \varsubsetneqq Q_{j_{0}}^{+}}}\left|Q_{j}\right| \leq 2^{n}\left|Q_{j_{0}}\right| .
$$

Therefore, since for every $j \in \Gamma_{\mu}, \mu<f_{Q_{j}^{+}} \leq 2 \mu$, we get

$$
\begin{gathered}
\sum_{m>m_{0}} \int_{\sigma_{m} \cap Q_{j_{0}}^{+}} f=\sum_{m>m_{0}}^{\infty} \sum_{\substack{j \in i_{m}: \\
Q_{j}^{+} \varsubsetneqq Q_{j_{0}}^{+}}} \int_{Q_{j}^{+}} f \\
\leq 2 \mu \sum_{m>m_{0}} \sum_{\substack{j \in i_{m}: \\
Q_{j}^{+} \varsubsetneqq Q_{j_{0}}^{+}}}\left|Q_{j}\right| \leq 22^{n} \mu\left|Q_{j_{0}}\right|<2^{n+1} \int_{Q_{j_{0}}^{+}} f .
\end{gathered}
$$

Thus, we have that

$$
\sum_{m=m_{0}+1}^{m_{0}+2^{n+2}} \int_{\sigma_{m} \cap Q_{j_{0}}^{+}} f<2^{n+1} \int_{Q_{j_{0}}^{+}} f .
$$

Then, there is an index $m, m_{0}+1 \leq m \leq m_{0}+2^{n+2}$, such that $\int_{\sigma_{m} \cap Q_{j_{0}}^{+}} f<\frac{1}{2} \int_{Q_{j_{0}}^{+}} f$, and since $\int_{\sigma_{m+1} \cap Q_{j_{0}}^{+}} f \leq \int_{\sigma_{m} \cap Q_{j_{0}}^{+}} f$, we have that, if $j_{0} \in m_{0}$,

$$
\int_{Q_{j_{0}}^{+} \cap \sigma_{m_{0}+2^{n+2}}} f<\frac{1}{2} \int_{Q_{j_{0}}^{+}} f
$$

which implies that

$$
\int_{Q_{j_{0}}^{+}-\sigma_{m_{0}+2^{n+2}}} f>\frac{1}{2} \int_{Q_{j_{0}}^{+}} f .
$$

From the last inequality and since $\mu<f_{Q_{j_{0}}^{+}}$, we get

$$
\frac{1}{\left|Q_{j_{0}}\right|} \int_{Q_{j_{0}}^{+}-\sigma_{m_{0}+2^{n+2}}} f>\frac{\mu}{2} .
$$

Now we will estimate $\sum_{j \in \Gamma_{\mu}} w\left(Q_{j}\right)$. We suppose $p>1$. From (3]), Hölder's inequality, and by condition $A_{p}^{+, d}\left(\mathbb{R}^{n}\right)$, we have

$$
\begin{aligned}
\sum_{j \in \Gamma_{\mu}} w\left(Q_{j}\right) & =\sum_{m=0}^{\infty} \sum_{j \in i_{m}} w\left(Q_{j}\right) \leq\left(\frac{2}{\mu}\right)^{p} \sum_{m=0}^{\infty} \sum_{j \in i_{m}} w\left(Q_{j}\right)\left(\frac{1}{\left|Q_{j}\right|} \int_{Q_{j}^{+}-\sigma_{m+2^{n+2}}} f\right)^{p} \\
& \leq\left(\frac{2}{\mu}\right)^{p} \sum_{m=0}^{\infty} \sum_{j \in i_{m}} w\left(Q_{j}\right)\left[v^{-\frac{1}{p-1}}\left(Q_{j}^{+}\right)\right]^{p-1} \frac{1}{\left|Q_{j}\right|^{p}} \int_{Q_{j}^{+}-\sigma_{m+2^{n+2}}} f^{p} v \\
& \leq \frac{2^{p} C}{\mu^{p}} \sum_{m=0}^{\infty} \int_{\sigma_{m}-\sigma_{m+2^{n+2}}} f^{p} v .
\end{aligned}
$$


(If $p=1$ we simply observe that

$$
\frac{w\left(Q_{j}\right)}{\left|Q_{j}\right|} \int_{Q_{j}^{+}-\sigma_{m+2^{n+2}}} f \leq \int_{Q_{j}^{+}-\sigma_{m+2^{n+2}}} f M^{-, d} w,
$$

and by condition $A_{1}^{+, d}\left(\mathbb{R}^{n}\right)$ we can obtain the previous estimate also in this case.) Then, by (4) and since

$$
\begin{aligned}
\sum_{m=0}^{\infty} \int_{\sigma_{m}-\sigma_{m+2^{n+2}}} f^{p} v & =\sum_{k=0}^{2^{n+2}-1} \sum_{m=0}^{\infty} \int_{\sigma_{2^{n+2} m+k}-\sigma_{2^{n+2}(m+1)+k}} f^{p} v \\
& =\sum_{k=0}^{2^{n+2}-1} \int_{\sigma_{k}} f^{p} v \leq 2^{n+2} \int_{\sigma_{0}} f^{p} v,
\end{aligned}
$$

we obtain the lemma.

Proof of Theorem 1.2. We will prove that $(a) \Rightarrow(b)$ since $(b) \Rightarrow(a)$ can been obtained, with simple changes, by a standard argument (e.g., see p. 390 in [2]).

Assume that $(w, v) \in A_{p}^{+, d}\left(\mathbb{R}^{n}\right)$. We will estimate

$$
w\left(\left\{x: M^{+, d} f(x)>\lambda\right\}\right)=w\left(\Omega_{\lambda}\right) .
$$

Recall that we can write $\Omega_{\lambda}=\bigcup_{j} Q_{j}$, where $f_{Q_{j}^{+}}>\lambda$.

For each non-negative integer $k$, we consider the subfamily of indexes

$$
c_{k}=\left\{j: 2^{k} \lambda<f_{Q_{j}^{+}} \leq 2^{k+1} \lambda\right\} .
$$

Then, we can apply Lemma 2.1 to the collection $\left\{Q_{j}\right\}_{j \in c_{k}}$, with $\mu=2^{k} \lambda$, getting

$$
\sum_{j \in c_{k}} w\left(Q_{j}\right) \leq \frac{2^{p+n+2} C}{\left(2^{k} \lambda\right)^{p}} \int_{\mathbb{R}^{n}} f^{p} v .
$$

Therefore

$$
\begin{gathered}
w\left(\Omega_{\lambda}\right)=\sum_{j} w\left(Q_{j}\right)=\sum_{k=0}^{\infty} \sum_{j \in c_{k}} w\left(Q_{j}\right) \\
\leq \sum_{k=0}^{\infty} \frac{2^{p+n+2} C}{\left(2^{k} \lambda\right)^{p}} \int_{\mathbb{R}^{n}} f^{p} v=\frac{2^{p+n} 8 C}{\lambda^{p}} \int_{\mathbb{R}^{n}} f^{p} v,
\end{gathered}
$$

which proves that $(a) \Rightarrow(b)$.

Proof of Theorem 1.3. Fix an integrable and non-negative function $f$. We know that

$$
\left\{x: M^{+, d} f(x)>4^{-n} \lambda\right\}=\bigcup_{j} Q_{j},
$$

where $\left\{Q_{j}\right\}_{j}$ is a disjoint collection of dyadic cubes $\left\{Q_{j}\right\}_{j}$ such that for each $j$, $f_{Q_{j}^{+}}>4^{-n} \lambda$. We will prove that

$$
\left\{x: N^{+} f(x)>\lambda\right\} \subset \bigcup_{j}\left(Q_{j}\right)^{4,-} .
$$

If $x \in\left\{x: N^{+} f(x)>\lambda\right\}$ there exists $h>0$ such that $f_{Q_{x, h}^{\frac{1}{2}}}>\lambda$. Let $k$ be the integer such that $2^{k+1} \leq h<2^{k+2}$. Then, there exist at most $4^{n}$ dyadic cubes $R$ 
such that $R \cap Q_{x, h}^{\frac{1}{2}} \neq \emptyset$ and $|R|=2^{k n}$, and therefore one of them satisfies that $\int_{R} f \geq 4^{-n} \int_{Q_{x, h}^{r}} f$. Then, since $|R|=2^{k n} \leq\left|Q_{x, h}^{\frac{1}{2}}\right|$, we have that

$$
\frac{1}{|R|} \int_{R} f \geq \frac{4^{-n}}{|R|} \int_{Q_{x, h}^{r}} f \geq \frac{4^{-n}}{\left|Q_{x, h}^{\frac{1}{2}}\right|} \int_{Q_{x, h}^{\frac{1}{2}}} f>4^{-n} \lambda,
$$

and therefore the dyadic cube $R^{-} \subset\left\{x: M^{+, d} f(x)>4^{-n} \lambda\right\}$. Thus there exists a maximal dyadic cube $Q_{j}$ in the collection in (15) such that $R^{-} \subset Q_{j}$; thus $\left(R^{-}\right)^{4,-} \subset$ $\left(Q_{j}\right)^{4,-}$ and since $x \in\left(R^{-}\right)^{4,-}$ we obtain (6) .

Now, by condition $A_{p}^{+}\left(\mathbb{R}^{n}\right)$, we have that

$$
\begin{gathered}
\int_{\left(Q_{j}\right)^{4,-}} w\left(\int_{\left(Q_{j}^{+}\right)^{4,+}} v^{-\frac{1}{p-1}}\right)^{p-1} \leq C\left|Q_{j}\right|^{p}, \text { if } p>1, \text { and } \\
\frac{1}{\left|Q_{j}\right|} \int_{\left(Q_{j}\right)^{4,-}} w \leq C M^{-} w(x) \leq C v(x) \text { for a.e. } x \in Q_{j}^{+}, \text {if } p=1 .
\end{gathered}
$$

Thus, arguing as in the proof of Lemma 2.1 and taking into account the last inequalities, we can prove that

$$
\sum_{j: 2^{k-2 n} \lambda<f_{Q_{j}^{+}} \leq 2^{k-2 n+1} \lambda} w\left(\left(Q_{j}\right)^{4,-}\right) \leq \frac{C}{\left(2^{k} \lambda\right)^{p}} \int_{\mathbb{R}^{n}}|f|^{p} v .
$$

Then, following the reasoning as in the proof of Theorem 1.2 we can obtain the theorem.

Final remark. We have already observed that if $n>1$ the maximal functions $N^{+} f(x)$ and $M^{+} f(x)$ are not pointwise equivalents. However, we believe that it is possible to prove that if the pair of weights $(w, v)$ satisfies the condition $A_{p}^{+}\left(\mathbb{R}^{n}\right)$, then the maximal operator $M^{+}$is of weak type $(p, p)$ with respect to $(w, v)$.

I would like to thank the referee and P. Panzone for their helpful comments.

\section{REFERENCES}

[1] H. Aimar, L. Forzani and F. J. Martín-Reyes, On weighted inequalities for singular integrals, Proc. Amer. Math. Soc. 125 (1995), 2057-2064. MR1376747 (97i:42012)

[2] J. Garcia Cuerva, and J. L. Rubio de Francia, Weighted norm inequalities and related topics, North Holland, (1985).

[3] F. J. Martín-Reyes, New proofs of weighted inequalities for the one-sided Hardy-Littlewood maximal functions, Proc. Amer. Math. Soc. 117 (1993), 691-698. MR.1111435 (93d:42016)

[4] F. J. Martín-Reyes, On the one-sided Hardy-Littlewood maximal function in the real line and in dimensions greater than one, Fourier analysis and partial differential equations (Miraflores de la Sierra, 1992), 237-250, Stud. Adv. Math. CRC, Boca Raton, FL, 1995. MR 1330244 (96f:42023)

[5] F. J. Martín-Reyes and A. de la Torre, Two weight norm inequalities for fractional one-sided maximal operators, Proc. Amer. Math. Soc. 117 (1992), 691-698. MR.1110548 (94b:42010)

[6] F. J. Martín-Reyes, P. Ortega-Salvador, and A. de la Torre, Weighted inequalities for one-sided maximal functions, Trans. Amer. Math. Soc. 319 (1990), 517-534. MR0986694 (90j:42047)

[7] B. Muckenhoupt, Weighted inequalities for the Hardy maximal function, Trans. Amer. Math. Soc. 165 (1972), 207-226. MR0293384 (45:2461)

[8] L. de Rosa and C. Segovia, Weighted $H^{p}$ spaces for one sided maximal functions, Contemporary Math. 189 (1995), 161-183. MR1347012 (96k:42027) 
[9] L. de Rosa and C. Segovia, Dual spaces for one-sided weighted Hardy spaces, Rev. Un. Mat. Arg. 40 (1997), 49-71. MR1616750 (99h:42039)

[10] E. Sawyer, Weighted inequalities for the one-sided Hardy-Littlewood maximal functions, Trans. Amer. Math. Soc. 297 (1986), 53-61. MR.0849466 (87k:42018)

Departamento de Matemática, Universidad Nacional del Sur, Avenida Alem 1253, Bahía Blanca, Buenos Aires, Argentina

E-mail address: sombrosi@uns.edu.ar 